\title{
Profili bioetici dell'utilizzo del placebo in medicina
}

\author{
Pasquale Giustiniani ${ }^{1}$
}

1 Docente di Bioetica, Facoltà di Giurisprudenza della Seconda Università degli Studi di Napoli

\begin{abstract}
Placebo is defined as an inert substance or inactive treatment. It is usually used in clinical trials as a control and its effect is so significant that the "gold standard" of research design is the doubleblind, placebo-controlled study. The question thus arises whether placebos should be used therapeutically.

In the proposed case, the deceptive use of placebo on a teenager in USA allowed his mother to bring to court a doctor and three nurses for malpractice. This paper, from the bioethics point of view, discusses the clinical and ethical perspectives of the placebo use in clinical practice.
\end{abstract}

Keywords: bioethics, placebo, pain medicine, malpractice

Bioethics issues of the placebo use in medicine.

Pratica Medica \& Aspetti Legali 2008; 2(1): 29-37

\section{INTRODUZIONE}

Benché già una risoluzione del Parlamento europeo del 1997 enunci chiaramente il dovere di tutelare la libertà di scelta dei singoli utenti rispetto alle varie terapie presenti sul "mercato" - sia convenzionale che non - sono ancora numerose le resistenze, da parte di esperti e pazienti, di fronte all'eventualità della somministrazione di medicinali e farmaci che non risultino del tutto accreditati e, soprattutto, non garantiti da prove di efficacia e di sicurezza.

$\mathrm{Ci}$ si fida giustamente di prodotti immessi nell'ordinaria prassi terapeutica a seguito di una lunga e controllata sperimentazione scientifica ufficiale, peraltro previamente autorizzata dai Comitati Etici. Ecco anche perché, di fronte a certi successi ottenuti da medicine alternative o non convenzionali - che, per quanto accreditate, sono tuttavia escluse dal novero dei Livelli Essenziali di Assistenza (LEA) nel nostro Servizio Sanitario Nazio- nale ${ }^{1}$-, non manca oggi chi parla subito di "effetto placebo". L'espressione assume, tuttavia, risonanze denigratorie, quasi a voler indicare che qualunque esito positivo ottenuto con sostanze farmacologicamente inattive al di fuori di una prassi terapeutica basata sulla scienza e sull'evidenza sia sempre da spiegare come un momentaneo stato di miglioramento della sintomatologia, piuttosto

\footnotetext{
${ }^{1}$ Dai LEA, di cui al d.P.C.M 29 novembre 2001 e successiva Legge n. 289/2002, risultano escluse: agopuntura fatta eccezione per le indicazioni anestesiologiche - fitoterapia, medicina antroposofica, medicina ayurvedica, omeopatia, chiropratica, osteopatia nonché tutte le altre discipline non espressamente citate, e le medicine non convenzionali. Non manca in merito il contenzioso tra Regioni e Stato. La Corte Costituzionale, dopo la precedente sentenza $n .88 / 03$, è tornata sulla problematica nella sentenza n. 134/06, quasi in contemporanea con il varo del nuovo Piano sanitario nazionale 2006-2008, il cui paragrafo 3.2 è dedicato, appunto, alla garanzia e all'aggiornamento dei $L E A$
} 
che come un'effettiva guarigione. Anziché al farmaco non convenzionale o alla sostanza inerte, si dice, qualunque eventuale risultato benefico ottenuto sarebbe da ascrivere, tutt'al più, al caloroso, amichevole e rassicurante modo di porsi del terapeuta, ovviamente anch'esso, a sua volta, senz'alcuna convalida empirica di efficacia relativamente agli effetti. Infondato, quindi, supporre - come invece insinuava Henry Beecher già nel 1955 che i benefici sui pazienti trattati potrebbero non essere soltanto soggettivi e psicologici, ma verosimilmente anche oggettivi e fisici, insomma simili a quelli indotti da un farmaco "vero" dotato di attività specifica ${ }^{2}$ [1].

Oltre a screditare con troppa leggerezza tutto ciò che, in qualche modo, esuli dal sistema "standard" della prassi scientifica di tipo moderno (osservativa-sperimentale e, in definitiva, fisico-matematica) e a marginalizzare qualunque altro tentativo terapeutico non convenzionale, un giudizio affrettato sull'inutilità $o$, addirittura, sulla pericolosità di sostanze e prassi alternative, però, non nuoce soltanto al placebo ${ }^{3}$. A parte il fatto che quest'ultimo, come si vedrà in seguito, continua ad essere utilizzato nella medicina scientifica, un tale giudizio mostrerebbe come minimo di non tenere in debito conto un principio, di rilevanza costituzionale, ribadito anche da pronunciamenti del giudice delle leggi. Si allude alla "libertà di cura" del paziente, anche di fronte a proposte non allopatiche o non tradizionali, di cui non sia stato già provato l'effetto dannoso. Così, per quanto riguarda gli eventuali risultati, sarebbe troppo leggero omologare tout court al placebo e alla sua "capricciosità esplicativa" qualunque altra "medicina" praticata da millenni in culture non occidentali, misconoscendone vantaggi significativi riferiti dai soggetti che vi hanno fatto ricorso. Altrettanto leggero, inoltre, sarebbe sottovalutare il ruolo del "pensiero positivo", della fiducia, delle attese soggettive e delle credenze sull'evoluzione di una malattia.

$\mathrm{E}$ se, in nome della libertà di cura, quindi anche dell'eventuale non-cura o di una sostanza farmacologicamente inattiva, ma terapeuticamente non del tutto inefficace, si ottenesse almeno l'effetto benefico di una ri-apertura della discussione scientifica ed etica intorno alle capacità curative della "fiducia" e del contesto relazionale?

Un peculiare caso - benché verificatosi in un'altra cultura e in un altro sistema sanitario - ci può consentire, appunto, d'indugiare non soltanto sul-

\footnotetext{
2 Ringrazio il prof. Gianluigi Zeppetella (Ospedale San Sebastiano di Caserta) per questa preziosa indicazione

3 Su queste problematiche, cfr almeno Centro Interuniversitario di Ricerca Bioetica (a cura di). Etica della salute e "Terapie non convenzionali". Napoli: Giannini, 2002. Circa l'assimilazione dell'omeopatia ad acqua fresca o a un similplacebo, cfr ad esempio Goldacre B. Benefits and risks of homoeopathy. Lancet 2007; 370: 1672-3
}

la correttezza o coerenza dell'eventuale ricorso al placebo in vista del risultato del benessere, o almeno dell'attutimento del malessere in un paziente, ma anche di aprirci alla più vasta problematica della tutela della salute umana nella sua integralità.

\section{EFFETTI DEL PLACEBO E MALPRACTICE SANITARIA: UN CASO STATUNITENSE}

Un adolescente fu ricoverato in un ospedale a seguito di forti emicranie. Durante la degenza, un medico sostituì il trattamento protocollare, a base di analgesico oppiode, con un placebo (verosimilmente una soluzione salina). Il dolore del giovane paziente, non trattato in maniera convenzionale, regredì sufficientemente al punto che egli poté essere dimesso. Tuttavia la madre, una volta scoperto che sul proprio figlio era stato praticato un ingannevole uso del placebo, accusò il medico curante e tre infermieri professionisti di malpractice o scorrettezza professionale, in quanto avrebbero dovuto, per motivi deontologici, ricorrere all'uso del placebo soltanto in situazioni attentamente valutate oltre che, ovviamente, su specifica indicazione della scienza medica. Mentre il medico non fu sanzionato dagli organi di controllo interno, per gli infermieri fu invece avviato un procedimento del giudice amministrativo.

Sul piano meramente formale, il caso, oltre a risultare piuttosto inverosimile nel nostro orizzonte socio-sanitario, esibisce negli esiti stessi della vicenda alcuni chiari profili. In primo luogo, le rimostranze della madre sarebbero legittime e fondate se lei, in quanto "amministratrice di sostegno" del bene salute dell'adolescente, si fosse aspettata una proposta terapeutica e farmacologica "attiva”, ottenendone invece una mancata azione, peraltro neppure previamente notificata. A sua volta, l'insuccesso legale dell'azione promossa contro il medico evidenzia che non essendovi stata, da parte dello specialista, una positiva disposizione, ma soltanto l'indicazione per la somministrazione di una sostanza inerte, non esisteva fondamento per un'azione disciplinare o risarcitoria per malpractice attiva. A sua volta, il procedimento a carico degli altri professionisti non medici, oltre a segnalare il possibile contenzioso tra atti medici e atti non medici - oggi in via di emersione anche in Italia a seguito di curricola universitari per la preparazione degli infermieri - esplicita una certa tendenza degli organi di controllo: essi sembrano, infatti, tendere ad isolare gli atti - in questo caso, la somministrazione di una sostanza inerte anziché 
di una terapia efficace disponibile - puntualmente compiuti, piuttosto che aprirsi anche alla problematica teorica e olistica. In ogni caso, infatti, le forti emicranie - sintomatologia, questa, a rilevante componente neurologica e psico-relazionale regredirono al punto che poté essere autorizzata la dimissione del ragazzo. Come ben sanno coloro che studiano il complesso mondo delle cefalee e del dolore di varia natura e localizzazione (cefalea, dolore anginoso, artrite, mal di stomaco, ecc), tali patologie spingono oggi a tenere in maggior conto tutte le prassi mediche e non, farmacologiche e non, che aiutino a far regredire le reazioni emozionali e organiche $[2,3]$. Tutto quello che ha che vedere con le componenti psicogene ed emotive del disagio fisico, e non soltanto con esse, spinge a non porre mai in secondo piano le numerose vie, soltanto in parte fisio-chimiche, di reazione del paziente alle cure e alle prestazioni. Questo, di rimando, fa risaltare la rilevanza del contesto socio-sanitario, dei rapporti interpersonali, perfino dell'uso di preparati non tradizionali nel processo di assistenza e guarigione.

Se, ribadiamolo, il soggetto in situazione di malessere o di disagio, in cui il dolore è da considerare come un vero e proprio indicatore fenomenico o "avvisatore", non è mai riducibile alla sua sola componente fisio-chimica, anche la situazione di cura ne dovrebbe risultare modificata. Soprattutto, essa appare sempre meno riducibile alle sole costituenti protocollari, quali ad esempio l'uso di preparati di sintesi o di altre sostanze. Troppo numerose e complesse, infatti, sono le dimensioni di quel peculiare sistema fisio-psico-simbolico che è l'essere umano. Ecco perché il caso di "malasanità" americana, può utilmente assumere un valore euristico dal punto di vista bioetico.

\section{ALCUNI PROFILI DEL CASO}

Ci si può, così, domandare se l'eventuale ricorso a metodi che si pongano ora al di fuori dei protocolli standardizzati, ora al di fuori della medicina "ufficiale", siano davvero a priori incongrui rispetto al diritto di ogni persona a godere del proprio stato di salute.

Esiste pur sempre un diritto del paziente a scegliere e ottenere una qualunque terapia consapevolmente determinata come più utile e vantaggiosa al proprio benessere. Più in dettaglio, ci si potrebbe chiedere se un atto medico e, in genere, curativo, abbia come esclusiva indicazione terapeutica il ricorso a farmaci o altre pratiche con effetti biochimici sul paziente. Perché non assegnare - ovviamente sulla scia di un più ampio ripensamento dei rapporti tra mente e corpo - un maggiore rilievo all'interazione professionale degli operatori della salute e del contesto ospedaliero con tutte quelle componenti culturali, psicologiche, pedagogiche e simboliche dell'essere umano? Tutti questi, infatti, appaiono oggi nella pratica clinica sempre più dei fattori concomitanti in grado di provocare, indipendentemente dall'effettivo uso del placebo, un vero esito positivo non soltanto sulla vita di relazione del paziente, ma sulla sua stessa percezione della sofferenza.

A meno che non si decida di liquidare il tutto come un inutile slittamento di una discussione medica nel campo della filosofia della medicina, ci si può altresì chiedere se il miglioramento, non episodico e non momentaneo, nella percezione della malattia da parte del paziente trattato con placebo sia davvero da ritenere l'effetto di un comportamento colposo od omissivo da parte dei professionisti della salute.

Come la madre del ragazzo statunitense successivamente sostenne in sede di azione legale, non è del tutto certo che si siano configurate delle scorrettezze professionali, per esempio una mancata cura nei confronti di un soggetto malato, con l'aggravante dell'età adolescenziale. Posto il dato che, nel soggetto in questione, non si era comunque verificata un'accelerazione bensì una regressione della malattia, ovvero non si era registrato neppure un effetto "nocebo" (che, com'è noto, viene documentato come una delle possibilità collaterali negative della somministrazione del placebo $)^{4}$, l'astensione dalla somministrazione di farmaci è davvero da ritenere una condotta, se non proprio scorretta, almeno inopportuna? Probabilmente le condizioni di salute del ragazzo erano sembrate agli specialisti da riportare più a componenti emotive e psico-relazionali che a oggettive cause fisiologiche. Non essendovi stata alcuna comparazione controllata tra diversi prodotti e relativi effetti sulla regressione della patologia, non essendovi poi stata comparazione tra pazienti, non esistendo uno standard condiviso di valutazione degli effetti ottenuti, se ne dovrebbe, tuttavia, concludere che la dimissione sia stata decisa non per aver constatato motivi scientifici utili al progresso della conoscenza medica. Oltre agli aggravi di spesa sanitaria per la degenza inutile, resterebbe, in tal caso, aperta la questione circa la possibilità di dimettere un paziente a seguito di una sua semplice sensazione soggettiva di miglioramento.

\footnotetext{
${ }^{4}$ Insieme all'effetto nocebo (insorgenza di effetti negativi nel corso dell'uso del placebo in trial clinici), risulta abbastanza documentata l'enorme variabilità dei fattori che intervengono a determinare l'esito dell'intervento: per esempio, il metodo con il quale avvengono le registrazioni degli effetti, la modalità con la quale il paziente riceve le informazioni riguardanti la terapia, il contesto in cui avviene la prescrizione del farmaco, il rapporto medico-paziente, ecc
} 


\section{USI E ABUSI DEL PLACEBO}

Assai più noto nel campo delle medicine convenzionali, soprattutto all'interno dei trial clinici randomizzati (RCTs), il placebo consente ancor oggi di osservare, in alcuni casi e a determinate condizioni, degli effetti benefici sul piano scientifico e medico. Difatti, pur potendosi stabilire in maniera soddisfacente l'efficacia di certuni farmaci senza ricorrere al placebo, il corretto uso di questo prodotto appare in Italia ancora essenziale al fine di controllare l'oggettiva efficacia di certi medicamenti o pratiche mediche. Il placebo, in questo senso, costituisce ancora un "farmaco di controllo", quindi una pietra di paragone, per lo meno allo scopo di evitare suggestioni o interferenze nell'osservatore-ricercatore di fronte all'andamento di un determinato fenomeno patologico o al funzionamento di un farmaco nelle varie fasi della sua sperimentazione. Questo è particolarmente vero nel corso della cosiddetta III fase della sperimentazione, allorché il nuovo farmaco, o la nuova terapia sperimentale, vengono di norma comparati, appunto, con il miglior trattamento disponibile e, se è il caso, anche con un placebo. Tutto questo non avviene, però, senza discussioni. Come ha precisato nel 2001 il nostro Comitato Nazionale di Bioetica (CNB), l'uso eventuale di placebo, anche a motivo dei suoi effetti suggestivi o puramente psicologici, trova una qualche sua giustificazione soltanto nei trial clinici. Questi, infatti, «hanno una connotazione ben precisa in quanto costituiscono uno studio longitudinale in cui si impiegano uno o più gruppi di controllo rispetto al gruppo che subisce il trattamento, e in cui l'assegnazione a uno dei due bracci dello studio dei soggetti utilizzati per l'esperimento viene effettuata a caso (at random). Nel gruppo di controllo può essere prevista la somministrazione di un placebo ovvero di una usuale terapia standard. [...] Questi artifizi hanno lo scopo di evitare che nella valutazione dei risultati vi siano interferenze da parte dei pregiudizi del ricercatore il quale, in tal modo, può anche studiare gli effetti puramente psicologici, suggestivi, esercitati dal trattamento sul paziente di controllo" [4].

In una procedura per tentativi ed errori, quale rimane appunto una sperimentazione medica o farmacologica, e particolarmente in presenza di patologie che comportino sofferenze non soltanto di tipo neurologico e fisico, ma a componente psicologica e socio-relazionale, si dà, perciò, la possibilità di ricorrere a un placebo, comunque relegato alla funzione di sostanza senza effetti apprezzabili. Ovviamente sulla base di precise condizioni protocollari, anche se i difetti delle medie ottenute, come altresì le implicazioni residuali di terapie precedentemente praticate, non risulteranno mai ben valutabili. Lo scopo principale dell'uso, in ogni caso, rimane quello di tutelare la salute delle persone, entro un più vasto complesso di fattori, comprendenti la ripartizione delle competenze, il regime delle prestazioni, l'assetto organizzativo e la disciplina delle professioni sanitarie [5].

Ma che dire, come avviene nel caso esaminato, di fronte a eventuali decisioni di ricorso al placebo in un trattamento ordinario su un paziente che non sia stato preventivamente arruolato in un processo sperimentale e, per di più, sia degente in un reparto ospedaliero con sintomi che verosimilmente non sono già regrediti a seguito del ricorso alle ordinarie terapie disponibili? Oltre agli innegabili profili di deontologia medica e di etica medica, interrogativi simili sorgono in alcuni settori della medicina del dolore e della palliazione, oppure nel trattamento di malattie a prevalente o quasi esclusiva componente neurologica e psicologica (ad esempio, nel trattamento del Parkinson, della depressione, dei dolori e di altri disturbi osservati nei tossicodipendenti) [6].

Se, come sembrano dimostrare alcuni dati, il preteso effetto positivo del placebo risultasse comunque mediato da sostanze biochimiche che presiedono a determinati meccanismi di risposta del cervello umano, diventerebbero sempre più interessanti le indicazioni scientifiche di questa "non terapia". A parte il fatto che ogni effetto positivo di un farmaco può comunque esser considerato come la somma tra lo specifico esito farmacologico e il suo "effetto placebo", non sarebbero comunque risolti tutti i dubbi interpretativi. Per esempio, l'eventuale successo non permetterebbe, in ogni caso, di conoscere se l'efficacia sia da imputare al placebo, o ad effetti positivi di farmaci pregressi, oppure a un effetto simil-placebo (epressione che evoca la normale evoluzione psicosomatica della percezione nocicettiva del soggetto), e/o, forse più verosimilmente, agli effetti lenitivi indotti dal contesto socio-sanitario. Comunque si voglia rispondere, è almeno attendibile supporre che il contesto socio-sanitario, se opportunamente umanizzato, sia ben in grado di indurre nel paziente sensazioni, non esclusivamente soggettive, di benessere, quindi di migliorare la percezione del proprio stato di salute.

\section{PLACEBO, TERAPIA DEL DOLORE E RESPONSABILITÀ DEL MEDICO}

Il tipo di patologia evocata dall'episodio statunitense rinvia, come si accennava, a quel peculiare contesto di sintomatologie dolorose, in questi anni in continua evoluzione, che consentono l'emergere progressivo, nella pratica medica, del- 
la medicina del dolore e della terapia della sedazione e/o della palliazione. Il tutto è, a sua volta, da porre in correlazione con la problematica della "narrazione" delle malattie e delle sintomatologie dolorose da parte del soggetto. Il dolore/sofferenza, insomma, da fattore concomitante secondario nella lotta medica contro le malattie, diviene una componente rilevante di per sé, in grado di generare specifiche competenze disciplinari. Inoltre, data la genesi complessa, e non soltanto fisiologica, della nocicezione, la presenza di dolore, soprattutto in fase acuta, mette talvolta in crisi gli ordinari protocolli farmacologici, imponendo interventi in équipe e facendo assumere nuovo rilievo anche alle specialità non esclusivamente fisiologiche, ma psicologiche e perfino socio-culturali e spirituali.

Sempre più presente nel trattamento di casi patologici "ordinari" che esibiscono aspetti nocicettivi, la terapia del dolore non appare più relegata, come avveniva ancora fino a qualche decennio fa, a una prassi quasi ai limiti della medicina. Pur non essendo sempre finalizzata alla guarigione o alla cronicizzazione della percezione dolorosa, bensì soltanto al lenimento del sintomo sul piano biochimico e della sofferenza sul piano fisio-psichico, essa appare ormai una vera terapia, peraltro praticata non soltanto su pazienti irrimediabilmente compromessi o addirittura in situazione cosiddetta terminale. In tutti i casi in cui si ricorra a una terapia soltanto palliativa, si proclama comunque il valore, accanto alla prassi farmacologica e chirurgica, del prendersi cura e dell'accompagnare solidaristicamente il paziente. Com'è noto, si discute ancora su quale debba essere il settore scientifico principale di riferimento per un tal tipo di terapia che non riguarda uno specifico distretto fisiologico, dal momento che il dolore accompagna, in genere, le patologie di qualsiasi apparato o sub sistema umano. Resta comunque fermo, come affermava già il CNB nel 1995, che con la diffusione della palliazione si manifesta la ferma intenzione della medicina di non lasciar mai solo il paziente, ovvero di aiutarlo a vivere ogni sua esperienza nocicettiva nel modo più umano possibile, da ogni punto di vista. Ecco perché «volte primariamente ad alleviare il dolore in generale, e in particolare quello dei malati terminali, le cure palliative hanno allargato e continuano ad allargare il loro orizzonte e il loro ambito di azione e si presentano nel nostro tempo come uno dei campi in cui la moderna medicina manifesta la sua vocazione profonda di cura, in senso globale, quindi non solo fisico, ma anche psicologico e esistenziale, dei sofferenti» [7].

In sostanza, siamo di fronte a una medicina che punta su tutti quegli "elementi di contorno" - per certi aspetti analoghi a certi effetti indotti anche dal placebo - al fine di indurre nel soggetto la mitigazione delle pene, soprattutto di aiutarlo a rico- struire la sua percezione del dolore e attutire gli effetti collaterali negativi che qualunque terapia comunque comporta. Non è, questa, la via verso una medicina sempre meno "aggressiva" del malessere e sempre più "curante" nel senso ampio del termine, così come viene sostenuto nella cosiddetta "etica della cura"?

Quest'oggettivo allargamento dell'asse di riferimento, tipico della terapia medica palliativa, non ci sembra certo sufficiente a far maturare l'ipotesi di elevare anche il placebo al rango di terapia. Tuttavia, ci spinge almeno a prendere atto che, in ogni pratica medica, occorrerebbe mirare di più al complessivo senso lenitivo della terapia, al benessere-salute in senso olistico, su cui incidono non soltanto i protocolli e le sostanze, ma perfino la differente organizzazione del lavoro, la rimodulazione del modo scientifico di percepire il rapporto di cura come "alleanza terapeutica", le forme narrative espresse dal paziente nell'anamnesi, la diagnosi errata (i falsi positivi e negativi nella diagnostica preventiva sono all'ordine del giorno) e perfino una frase mal posta o distaccata da parte del professionista. Ne consegue che lo scopo di ogni terapia non potrebbe mai soltanto essere quello di compiere indagini strumentali finalizzate alla diagnosi o di prescrivere a tutti i costi presidi farmacologici (come sembra ancora reclamare la madre del ragazzo statunitense, che lamenta indirettamente la carenza di farmaci e terapie da lei giudicati più idonei), bensì quello di migliorare ulteriormente l'intero processo assistenziale a favore della persona, soprattutto nel controllo del suo dolore qualunque ne sia l'origine ${ }^{5}$.

Contigua a questa, è la discussione circa il peso della competenza specialistica del medico nel determinare i particolari protocolli terapeutici da osservarsi in presenza di patologie dolorose, come ad esempio le forti emicranie. Non si sottolineerà mai abbastanza il delicato compito del medico anestesista $\mathrm{o}$, laddove esistente, del palliativista o terapeuta del dolore, che dovrebbe garantire almeno la correttezza del metodo, se non proprio del risultato. Se bisogna evitare, soprattutto con i pazienti pediatrici, l'ostinazione in trattamenti da cui non si possa ragionevolmente attendere un beneficio per la salute del paziente e/o un miglioramento della sua qualità di vita, non si può abdicare al dovere di garantire comunque la sicurezza e il benessere dei pazienti. L'omissione di una terapia specifica, come sembra esser lamentato dalla

\footnotetext{
5 Su altro versante patologico, l'introduzione, nel nostro Sistema Sanitario, della struttura dell'hospice - le cui linee organizzative si propongono appunto di assicurare idonee terapie palliative ai pazienti che versano in "situazione terminale" - avvalora la rilevanza medica e scientifica del trattamento del dolore in ogni patologia, così come sancito, ad esempio, dalle linee guida per la realizzazione dell'ospedale senza dolore, di cui all'Accordo Stato-Regioni del 24 maggio 2001
} 
madre nel caso in discussione, costituisce, in definitiva, un indiretto rimprovero nei confronti dello specialista che abbia deciso per il ricorso a un placebo anziché per la migliore terapia accreditata disponibile. Il che ci apre ai rilevanti scenari contigui del risk management ${ }^{6}$ e alla problematica della proposta terapeutica competente. Lo specialista è, infatti, colui al quale sono affidati compiti di decisione "in scienza e coscienza" e non di semplice diligenza in analogia con il buon padre di famiglia. Perfino lo sforzo istituzionale di garantire, in caso di errore professionale, dei percorsi sempre più rapidi e snelli per il risarcimento del danno eventualmente arrecato ai cittadini, va inteso come una spinta alla qualificazione non paternalistica dei compiti e delle responsabilità del medico. Il che è tanto più valido se si tiene conto del perdurare di un'opinione pubblica in caccia di "malasanità" che, a sua volta, provoca la reazione della "medicina difensiva" e ritarda l'obiettivo della diagnosi clinica competente. Il ricorso audace, e deontologicamente non condivisibile, del medico statunitense a un placebo, può insomma fungere almeno da monito contro certe prassi che moltiplicano più del dovuto esami diagnostici e altri atti medici e terapeutici senza mai decidere. Siffatte procedure, infatti, sembrano talvolta utilizzate non tanto come utili supporti alla diagnosi, bensì al solo scopo di salvaguardare l'immagine e l'operato del professionista o, peggio ancora, di evitare successive aggressioni medico-legali o assicurative. D'altra parte, l'incremento, negli ultimi anni, delle azioni giudiziarie nei confronti degli operatori sanitari e delle strutture ospedaliere fa comunque supporre un'aumentata consapevolezza dei cittadini, desiderosi di una sanità più sicura e di un maggiore potere "contrattuale" rispetto agli erogatori dei servizi sanitari.

Nella stessa linea, la sollecita segnalazione di eventuali errori da parte degli operatori, pur nel rispetto della riservatezza dei dati, va assunta non tanto come indizio della volontà di mettere alla gogna il professionista che abbia errato, bensì di diffondere la conoscenza scientifica e, quindi, di prevenire ogni altra conseguenza indesiderata, aumentare la sicurezza del paziente, migliorare efficienza e qualità degli ospedali, ridurre $i$ costi aggiuntivi derivanti da errori, ridurre le spese di risarcimento e dei premi delle polizze assicurative.

${ }^{6}$ Si rammenterà che, con D.D. 20 febbraio 2006, presso la Direzione Generale della Programmazione sanitaria, dei livelli di assistenza e dei principi etici di sistema del Ministero della Salute, era stato istituito il "Gruppo di lavoro per la Sicurezza dei Pazienti”. Il Ministro della Salute Livia Turco, il 10 gennaio 2007, ha firmato il Decreto di istituzione del Sistema Nazionale di Riferimento per la Sicurezza dei Pazienti il quale, per garantire anche l'attività di Osservatorio per la Sicurezza dei Pazienti, opera sulla base di programmi annualmente approvati dal Ministro

\section{PRINCIPI ETICI ED ESIGENZE DEL PROGRESSO SCIENTIFICO: QUALE PRIMATO?}

Un ulteriore profilo del caso è quello relativo alla tutela della libertà di scelta delle terapie ritenute più idonee dal soggetto o dal suo rappresentante legale e/o amministratore di sostegno. In merito, la bioetica medica è passata dal principio di tutela del soggetto umano contro ricerche criminali, enunciato nel Codice di Norimberga dopo i crimini nazisti, a quello di beneficità del Belmont Report, fino alla Dichiarazione di Helsinki e successive revisioni, in cui è particolarmente in questione il principio del consenso informato, soprattutto nel caso di soggetti malati, immaturi o non competenti.

Proprio in merito al placebo, è stato ben osservato che, nel corso della quinta revisione della $\mathrm{Di}$ chiarazione di Helsinki, vi è stato «un confronto indubbiamente acceso, ma anche proficuo, tra chi sostiene che i principi etici debbano essere seguiti anche a costo di ostacolare il progresso scientifico e chi ritiene che debbano prevalere valutazioni maggiormente sfumate» [8]. Se è il paziente, infatti, che deve comunque autodeterminarsi alla scelta di tale o talaltra terapia proposta dallo specialista, anche eventualmente non convenzionale, si suppone nel medico la previa ponderata valutazione di tutte le migliori terapie disponibili, quindi anche la massima restrizione di alternative non sicure, e il placebo è tra queste. In merito, si tratta pur sempre di bilanciare la libera determinazione della persona, che potrebbe essere tuttavia compromessa dalla maggiore/minore gravità delle possibili conseguenze in caso di mancato trattamento sanitario, e una proposta terapeutica che domanda di accreditarsi scientificamente, non senza la previa valutazione circa l'eventuale danno che il paziente riceverebbe a seguito di un non-trattamento o di un trattamento non del tutto accreditato.

A partire dagli anni Novanta del XX secolo, si registra in letteratura una generalizzata contrarietà all'utilizzo dei placebo in medicina, come si vede ad esempio proprio alla fine di quegli anni, nella feroce critica rivolta perfino a certi trial clinici che, volendo ridurre il rischio di trasmissione perinatale di HIV, diedero luogo a comportamenti differenti su malati occidentali e malati dei Paesi più poveri, per i quali si era appunto fatto ricorso a un placebo al posto della terapia retrovirale standard.

Listanza etica che sovrintendeva a tale controversia è da identificare nella volontà, condivisibile, di garantire pienamente la salute e il benessere delle persone già in fase di sperimentazione, senza distinzione di ceto sociale, di etnia o di cultura. 
Le indicazioni della Convenzione di Oviedo sui Diritti dell'uomo e la biomedicina ${ }^{7}$ sono perentorie. Il dovere di salvaguardare i diritti dell'uomo e la dignità della vita umana a seguito delle nuove applicazioni della biologia e della medicina spinse perfino il nostro $\mathrm{CNB}$, nel corso dell'esame del protocollo applicativo della Convenzione, a notare un'impostazione non del tutto coerente tra gli orientamenti espressi nella bozza di testo e i punti precedentemente stabiliti, nel quadro della Comunità Europea, dalle "Linee guida per la buona

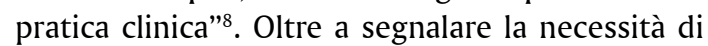
prevedere tutele per le persone non appartenenti ai Paesi firmatari del protocollo, il CNB riteneva assodato che l'eventuale uso di placebo, quando esistano metodi alternativi di trattamento di provata efficacia, debba considerarsi una patente violazione: «is a violation of the ethical code and is not in the interests of the patient. This cannot even be justified by reference to the interests of science and society» [9]. La formulazione della dichiarazione di Helsinki ${ }^{9}$ apparve in sostanza più felice dal punto di vista etico al punto che, in un successivo parere del CNB [10] i 32 principi di Helsinki saranno puntualmente ripresi per proporre più ampie riflessioni. In dettaglio, il CNB continuò a collocare il placebo all'interno della sperimentazione detta allora "non-terapeutica", la quale "ha lo scopo essenziale di verificare determinate ipotesi scientifiche senza tuttavia alcun beneficio diretto per il paziente o per il volontario sano che accetta di partecipare».

In sintesi, se ne può concludere che la discussione sul placebo, piuttosto che sui suoi eventuali effetti benefici e sui suoi effetti nocebo, nella nostra cultura tende elettivamente a fissarsi sul non uso. Prevalgono le considerazioni a salvaguardia del diritto del soggetto a ottenere il trattamento migliore attualmente disponibile. Su questa linea, ad esempio, si è mosso, in linea con il Parlamento europeo e il Consiglio, il Ministero italiano della Sanità [11], che ha imposto, già dalla metà degli anni Novanta del XX secolo, di evitare danni e abusi sui pazienti che si sottopongano a sperimentazione, valutando il peso del rischio di un danno

\footnotetext{
${ }^{7}$ Convenzione del Consiglio d'Europa sui diritti dell'uomo e sulla biomedicina, fatta a Oviedo il 4 aprile 1997, resa esecutiva con la legge di autorizzazione alla ratifica 28 marzo $2001, n$. 145 , però non ancora ratificata dallo Stato italiano. Cfr, più specificamente, Comitato direttivo per la bioetica del Consiglio d'Europa (CDBI), 29 luglio 1999 (Doc. CDBI-CO-GT2/99/10)

8 Adottate dalla Agenzia europea per la valutazione dei farmaci (CPMP/ICH/1995)

9 Dichiarazione di Helsinki I, approvata dalla XVIII Assemblea Medica Mondiale (1964) e quindi emendata periodicamente negli anni, in occasione dei successivi incontri assembleari
}

iatrogeno diretto o indiretto che potrebbe essere provocato per omissione di cure in soggetti trattati con solo placebo [12].

Quanto all'età pediatrica, si rammenti che, ad esempio, la Conferenza Stato-Regioni del 20 maggio 2004 relativamente agli studi clinici pediatrici di fase III e IV, pur prevedendo la possibilità d'inserimento di adolescenti (età 11-18) in casistiche riguardanti l'età adulta, ribadiva che gli studi debbono essere comunque condotti nel rispetto delle buone pratiche pediatriche e controllati verso la migliore terapia esistente e disponibile, con la precisazione che "studi non controllati sono ammessi solo quando non esiste trattamento e sono in questo caso da preferire agli studi verso placebo» [13].

Inoltre, se il Decreto Legislativo 24 aprile 2006, n. 219 [14] definisce come medicinale sperimentale anche una forma medicinale di placebo saggiato come medicina sperimentale o come controllo in una sperimentazione clinica, invece gli studi che, pur riguardando un farmaco, sono ritenuti non sperimentali, ma osservazionali, non ammettono mai l'eventualità del placebo. Su tutte queste implicazioni, del resto, non mancherà la puntuale vigilanza, ma anche il dibattito, da parte dei Comitati Etici ${ }^{10}$.

In sintesi su questo punto, non è prevedibile in Italia l'uso del placebo in situazione ospedaliera ordinaria, con il relativo coinvolgimento dei dirigenti di II livello e degli infermieri professionali, nonché dei Comitati Etici ${ }^{11}$.

\section{IN CONCLUSIONE}

Si potrebbe continuare a lungo. Ma in prospettiva conclusiva, si preferisce qui segnalare soltanto qualche spunto per l'ulteriore dibattito.

In primo luogo, la discussione relativa al rispetto dell'autodeterminazione del paziente in qualunque processo terapeutico - tema questo che, probabilmente, è ormai diventato qualcosa in più del consenso informato - in particolare nel caso

\footnotetext{
${ }^{10}$ In Italia il settore dei pazienti coinvolti nelle sperimentazioni cliniche di tipo interventistico è regolamentato dal DL 211/2003, che attua la Direttiva 2001/20/CE del Parlamento europeo e del Consiglio. Le Linee guida ministeriali per la classificazione e conduzione degli studi osservazionali sui farmaci, approvate con determinazione dell'Agenzia Italiana del Farmaco (AIFA), nell'agosto del 2007, ricordano l'importanza di analizzare previamente la qualità delle cure, dei profili assistenziali e delle relative ricadute economiche

${ }^{11}$ La specifica normativa italiana sui Comitati etici è stata adottata con DM 12.5.2006
} 
di pazienti incompetenti e in situazione di dolore. L'uso del placebo, o anche il ricorso a nessun trattamento qualora non esistano una provata profilassi, una diagnosi o un metodo terapeutico, non potrebbero essere mai subordinati a degli assoluti e scientificamente validi motivi metodologici del professionista. Se, almeno sul piano bioetico, comincia a profilarsi la discussione circa l'eventuale correttezza metodologica di un trattamento con placebo, deciso peraltro dall'acume del medico ben in grado di districarsi fondatamente tra i migliori trattamenti disponibili, va ricordato che la nostra giurisprudenza si muove diversamente, facendo prevalere la libera adesione del paziente. Infatti, "la correttezza o meno del trattamento non assume alcun rilievo ai fini della sussistenza dell'illecito per violazione del consenso informato, essendo del tutto indifferente ai fini della configurazione della condotta omissiva dannosa e dell'ingiustizia del danno, la quale sussiste per la semplice ragione che il paziente, a causa del deficit di informazione, non è stato messo in condizione di assentire al trattamento sanitario con una volontà consapevole delle sue implicazioni» [15]. La determinazione del paziente resta, insomma, un a priori, ovvero un presupposto di liceità di qualunque trattamento medico e chirurgico. Persiste un vero e proprio dualismo, quindi non unilaterale, nel processo di elaborazione di una decisione medica: medico che deve informare in ordine alla diagnosi e alle possibilità terapeutiche, e paziente, o suo legale rappresentante, che deve essere posto in grado di accettare o rifiutare i trattamenti prospettati.

A sua volta, sul piano della problematica dell'efficacia, l'orientamento che scoraggia l'uso di placebo negli stessi trial clinici è da collegare a un indirizzo costante, soprattutto alla fine del XX secolo, che ritiene più interessante testare l'efficacia di una nuova sostanza medicinale con quella di un medicinale già applicato e di cui sia noto il valore terapeutico, anziché con quella del "capriccioso" placebo ${ }^{12}$. Tuttavia, non mancano coloro che si domandano se il parametro dell'efficacia resti ancora l'unico da tener presente e se non convenga anche ricorrere al placebo per ottenere, se non proprio la regressione della patologia o il sostegno in corso di sofferenza, almeno delle vantaggiose informazioni su altri parametri incidenti nella costruzione soggettiva della patologia, quali il meccanismo d'azione, gli effetti collaterali, la via di somministrazione, l'interazione con altri farmaci e con il contesto interpersonale in ambiente sanitario, il costo.

${ }^{12}$ Cfr per esempio Direttiva 2001/83/CE del Parlamento europeo e del Consiglio del 6 novembre 2001 recante un codice comunitario relativo ai medicinali per uso umano, $n$. $F 1$

\section{BIBLIOGRAFIA}

1. Beecher HK. The powerful placebo. JAMA 1955; 159: 1602-6

2. Nestvold K, Kloster R, Partinen M, Sulkava R. Treatment of acute migraine attack: naproxen and placebo compared. Cephalalgia 1985; 5: 115-9

3. Hering R, Kuritzky A. Sodium valproate in the prophylactic treatment of migraine: a double-blind study versus placebo. Cephalalgia 1992; 12: 81-4

4. Comitato Nazionale di Bioetica. Scopi, limiti e rischi della medicina. 14 dicembre 2001

5. Cilione G. Diritto sanitario. Rimini: Maggioli editore, 2003

6. De la Fuente-Fernández R, Schulzer M, Jon Stoessl A. The placebo effect in neurological disorders. Lancet Neurology 2002; 1: 85-91

7. Comitato Nazionale di Bioetica. Questioni bioetiche relative alla fine della vita umana. 14 luglio 1995

8. Rosmini F. Punti di vista a confronto per la V revisione della Dichiarazione di Helsinki. Ann Ist Super Sanità 2002; 38: 169-74

9. Opinion of the National bioethics Committee on the European Protocol in biomedical research, 19 novembre 1999

10. Comitato Nazionale di Bioetica. Scopi, limiti e rischi della medicina. 14 dicembre 2001

11. Decreto 15 luglio 1997 e Linee-Guida di Buona Pratica Clinica per l'esecuzione delle sperimentazioni cliniche dei medicinali. Unione Europea, 1996

12. Direttiva n. 2001/204, aprile 2001, entrata in vigore il I maggio 2001 (cfr Gazzetta Ufficiale n. L 311, 28.11.2001, pp. 67128)

13. Conferenza Stato Regioni, seduta del 20 maggio 2004, n. 2.8.4

14. Gazzetta Ufficiale n. 142, 21.6.2006 in attuazione della Direttiva 2001/83/CE e successive, nonché Direttiva 2003/94/CE

15. Cassazione civile, sez. III, sentenza n. 5444 del 14 marzo 2006 


\section{PER APPROFONDIMENTI}

- Kissel P, Barrucand D. Placebos et effect placebo en medicine. Paris: Masson et C.ie Editeurs, 1964

- Fish JM. Placebo therapy, San Francisco-Washington-London: Jossey Bass, 1973

- Joyce CRB. Placebos and other comparative treaments. Br J Clin Pharmachol 1982; 13: 313-8

- Harrington A. (a cura di). The placebo effect. An interdisciplinary exploration. Cambridge-London: Harvard University Press, 1997

- Brody H. Per una filosofia della guarigione. Scienza ed etica dell'effetto placebo. Milano: Franco Angeli, 1998

- Moerman DE. Meaning, Medicine and the placebo effect. Cambridge: Cambridge University Press, 2002

- Moerman DE, Jonas WB. Deconstructing the placebo effect and finding the meaning response. Ann Intern Med 2002; 136:471-6

- $\quad$ Rich BA. A placebo for the pain: a medico-legal case analysis. Pain Medicine 2003; 4: 366-72

- Dobrilla G. Placebo e dintorni. Breve viaggio tra realtà e illusione. Roma: Il pensiero scientifico, 2004

- Lombardi Ricci M, Leone S. (a cura di). La fiducia che guarisce. Luso del placebo tra scienza ed etica. Acireale: ISB, 2006

- $\quad$ Rich BA. What is the place for placebo in the management of psychogenic disease? Lim and Seet J R Soc Med 2007; 100: 60-1

- Lipton BH. La biologia delle credenze. Come il pensiero influenza il DNA e ogni cellula. Diegaro di Cesena: Macro, 2007

\section{CORRESPONDING AUTHOR}

Prof. Pasquale Giustiniani, e-mail: pgiustiniani@libero.it 